\title{
Commercial Silicon-on-Insulator (SOI) Wafers as a Versatile Substrate for Laser Desorption/Ionization Mass Spectrometry
}

\author{
Shin Hye Kim, ${ }^{1,2}$ Jeongkwon Kim, ${ }^{2}$ Dae Won Moon,,${ }^{1,3}$ Sang Yun Han ${ }^{1}$ \\ ${ }^{1}$ Center for Nano-Bio Technology, Korea Research Institute of Standards and Science, Daejeon 305-340, Republic of Korea \\ ${ }^{2}$ Department of Chemistry, Chungnam National University, Daejeon 305-764, Republic of Korea \\ ${ }^{3}$ Daegu Gyeongbuk Institute of Science and Technology, Daegu 711-873, Republic of Korea
}

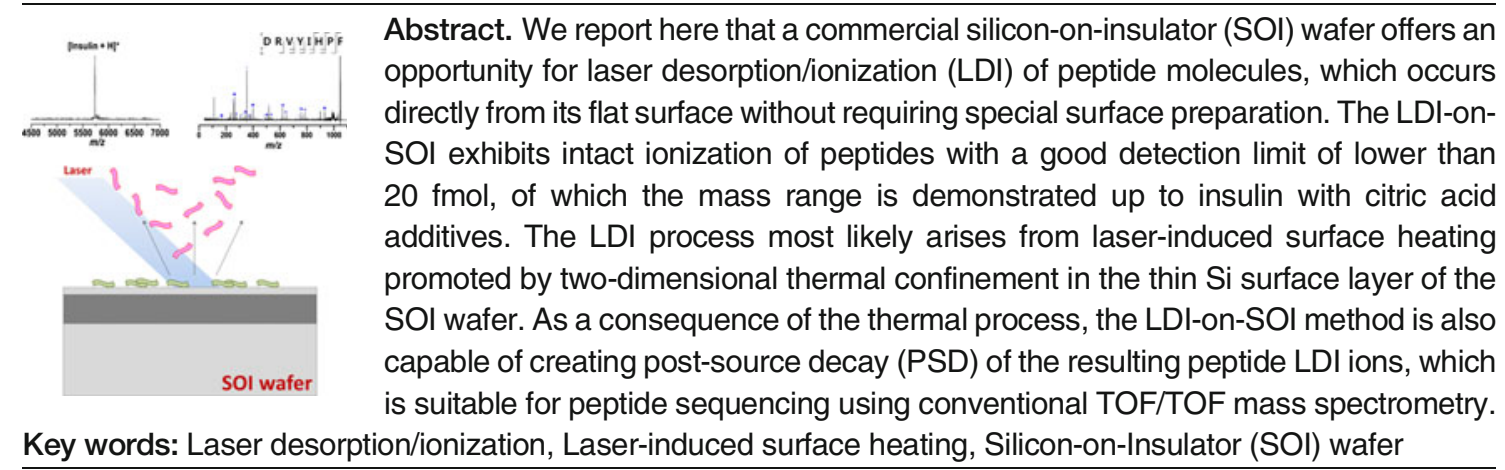

Received: 8 August 2012/Revised: 29 October 2012/Accepted: 4 November 2012/Published online: 19 December 2012

\section{Introduction}

I $\mathrm{n}$ the pursuit of new ionization methods, laser desorption/ ionization (LDI) has been the subject of extensive studies in recent years [1]. Various nanostructured surfaces have been successfully demonstrated for LDI of thermally labile molecules, with examples that include nanostructured $\mathrm{Si}$ surfaces (DIOS: desorption/ionization on porous Si) [2, 3], titania nanotube arrays [4], nanostructured gold thin films [5], HgTe nanostructures [6], and many more. In previous studies, the use of the surface nanostructures featured a common strategy in the creation of efficient LDI on the surfaces, although the role of the nanostructures involved in the LDI process has not yet been fully understood.

However, a few cases of LDI occurring on flat surfaces have also been reported. For example, it was recently found that the flat surfaces of amorphous $\mathrm{Si}$ (a-Si) [7] and tungsten silicide $\left(\mathrm{WSi}_{\mathrm{x}}\right)$ [8] yielded pronounced LDI of peptide molecules without assistance of surface nanostructures. As LDI on flat surfaces avoids the utilization of nanostructures that necessitates well-controlled surface fabrication, the use of flat surfaces makes it simpler, easier, and more reproducible to prepare uniform target surfaces. Despite many successful demonstrations, most LDI surfaces, either nano-

Correspondence to: Sang Yun Han; e-mail: sanghan@kriss.re.kr structured or flat, have been special and prepared in-house. This has limited the availability of the surfaces and, thus, their wider use and further development.

In this note, we report that the flat $\mathrm{Si}$ surfaces of commercial silicon-on-insulator (SOI) wafers provide an opportunity for LDI as well as post-source decay (PSD) without requiring special surface treatments. The pronounced LDI and PSD from SOI wafers are also discussed as being most likely promoted by a thermal mechanism (e.g., laser-induced surface heating). The use of commercially available wafers will be beneficial because it provides accessible LDI surfaces for those who seek to investigate the LDI process itself and also to develop further applications using matrix-free mass spectrometric monitoring.

\section{Materials and Methods}

Bradykinin 1-5 (monoisotopic mass $=572.3 \mathrm{Da}$ ), bradykinin 1-7 (756.4 Da), angiotensin II (1045.5 Da), substance P (1346.7 Da), melittin (2844.8 Da), insulin chain b oxidized (3493.6 Da), and insulin (5729.6 Da) were purchased from Sigma-Aldrich Korea (Suwon, Korea). 2,5-Dihydroxybenzoic acid (2,5-DHB), trifluoroacetic acid, acetic acid, ammonium hydrogen citrate (dibasic), and citric acid were also obtained from Sigma-Aldrich. HPLC-grade isopropy- 
lalcohol (IPA), acetonitrile (ACN), and methanol (MeOH) were purchased from Merck (Seoul, Korea). The reagents were used without further purification. The SOI wafers with $50 \mathrm{~nm}$ and $100 \mathrm{~nm}$ thick Si layers (8-inch diameter) were purchased from Nano Innovation Factory, Inc. (Suwon, Korea), of which the base oxide layers were nominally $200 \mathrm{~nm}$ thick.

\section{LDI-on-SOI Mass Spectrometry}

The SOI chips were cleaned via sonication in $100 \%$ IPA for $30 \mathrm{~s}$, washed in deionized water (Milli-Q; Millipore Korea, Seoul, Korea), and then dried under a gentle flow of nitrogen. The chips were further cleaned using a superpiranha solution $\left(\mathrm{H}_{2} \mathrm{O}_{2}, \mathrm{HNO}_{3}\right.$, and $\mathrm{H}_{2} \mathrm{SO}_{4}$ in a 10:1:6 volume ratio), rinsed thoroughly with deionized water and allowed to dry under a nitrogen stream.

For the typical LDI experiments, $0.2 \mu \mathrm{L}$ of $10 \mu \mathrm{M}$ peptide solution in $10 \% \mathrm{MeOH}$ in water $(2 \mathrm{pmol})$ was loaded on the SOI wafer chips. After drying under ambient conditions, the LDI experiments were performed using MALDI-TOF/TOF mass spectrometers (Autoflex III and Autoflex speed series; Bruker Daltonics, Bremen, Germany) equipped with a $355 \mathrm{~nm}$ UV laser. To study the effects of the citric acid additives, the sample solution was prepared in $60 \% \mathrm{ACN}$ in water containing $10 \mathrm{mM}$ citric acid. In the MALDI experiments, 2,5-DHB was used as the matrix. The PSD spectra were obtained using a MALDI-TOF/TOF mass spectrometer operated in LIFT-TOF/TOF mode [9].

\section{Results and Discussion}

\section{LDI of Peptides on SOI Wafers Via Laser-Induced Surface Heating}

For the LDI mechanism, laser-induced surface heating has been discussed as one of the main driving forces that induces intact desorption of thermally labile molecules from nanostructured surfaces. In the thermal mechanism, the surface nanostructures may act as heat conduction barriers. This can create a transient increase in the surface temperature up to as much as $1000 \mathrm{~K}$ by trapping the absorbed laser energy near the surface $[10,11]$. Such rapid surface heating is known to promote the intact desorption of thermally labile molecules from the early field desorption mass spectrometry [12]. Very recently, the thermal aspect involved in the LDI process has been further supported by pronounced LDI of peptides from the flat surfaces of a-Si and $\mathrm{WSi}_{\mathrm{x}}[7,8]$. The thermal properties of a-Si and $\mathrm{WSi}_{\mathrm{x}}$, such as a very low thermal conductivity $(\mathrm{K})$ and a very high heat capacity $\left(\mathrm{C}_{\mathrm{p}}\right)$, respectively, make the surfaces to be amenable to laser-induced surface heating, which may replace the needs of the presence of surface nanostructures for LDI of thermally labile molecules from the surfaces.

In this study, another possibility was explored for the LDI process based on the thermal mechanism. First, the possibility of laser-induced surface heating by two-dimensional heat confinement was considered; this occurs when the absorbed energy is trapped in the thin surface layer supported by a thermal insulator material with a lower thermal conductivity (K) (i.e., $\mathrm{K}$ (insulator) $<<\mathrm{K}$ (surface layer). The presence of underlying insulator material may provide an effective heat diffusion barrier, which enables the energy trapping within the thin surface layer. For the surface heating, the thickness of the surface layer may need to be smaller than the depth of the conductive heating $(D=[\mathrm{K} t /$ $\left.\mathrm{C}_{\mathrm{p}} \pi\right]^{1 / 2} ; t$ is the laser pulse width) of the layer material [7].

In order to verify this postulation, LDI on SOI wafers was explored. The SOI wafer possesses a layered structure of $\mathrm{Si}$ (thin surface layer) $-\mathrm{SiO}_{2}$ (thermal insulator; buried oxide layer) - Si substrate (handle wafer), which has provided an important platform for modern semiconductor technology [13]. The insulator layer in the SOI wafers $\left(\mathrm{SiO}_{2}\right)$ has a significantly lower thermal conductivity $\left(\mathrm{K}=1.3 \mathrm{Wm}^{-1} \mathrm{~K}^{-1}\right)$ than that of bulk Si $\left(150 \mathrm{Wm}^{-1} \mathrm{~K}^{-1}\right)$. In this study, SOI wafers with a $50 \mathrm{~nm}$ or $100 \mathrm{~nm}$ thick Si layer bonded on top

(a)
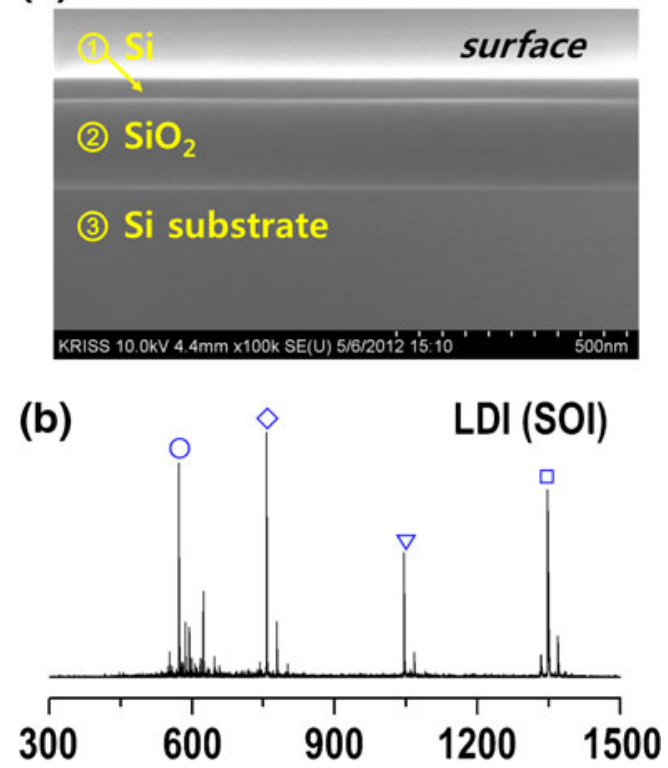

(c)

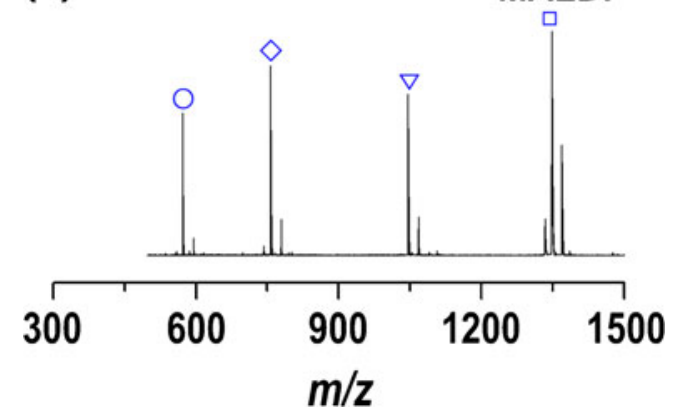

Figure 1. (a) SEM image (cross section) of the SOI wafer: (1) $\mathrm{Si}$ layer (50 nm thick), (2) buried oxide layer $\left(\mathrm{SiO}_{2}, 200 \mathrm{~nm}\right.$ thick), (3) Si wafer (handle wafer). (b) LDI and (c) MALDI mass spectra for the peptide mixture containing bradykinin 1-5 (O), bradykinin 1$7(\diamond)$, angiotensin II ( $\nabla)$, and substance P ( $\square)$ (2 pmol each) 
of $200 \mathrm{~nm}$ thick buried oxide were used (Figure 1a). The thickness of the Si layer was chosen to be smaller than the conduction depth $(D)$ of $450 \mathrm{~nm}$ for $\mathrm{Si}$, which was estimated using the literature values of $\mathrm{K}=150 \mathrm{~W} \mathrm{~m}^{-1} \mathrm{~K}^{-1}$ and $\mathrm{C}_{\mathrm{p}}=$ $1.67 \mathrm{~J} \mathrm{~cm}^{-3} \mathrm{~K}^{-1}$ for a laser pulse width of $t=7 \mathrm{~ns}$.

The results of LDI on the SOI wafers are presented in Figure $1 \mathrm{~b}$. As demonstrated in the LDI mass spectra, the irradiation of the UV laser on the SOI wafers induced intact desorption/ionization of peptide molecules from the Si surfaces, of which the mass spectrum is similar to that obtained using the MALDI method (Figure 1c). It is to be noted that LDI of thermally labile molecules from the flat surfaces of bare $\mathrm{Si}$ has never been reported without surface nanostructures [2, 3], surface coating [7], and buried insulator layers of this study. Although the intact desorption of thermally labile molecules from the SOI surfaces can be explained by laser-induced thermal desorption, the ionization mechanism (i.e., the chargerequiring step) in the LDI process still requires a further study.
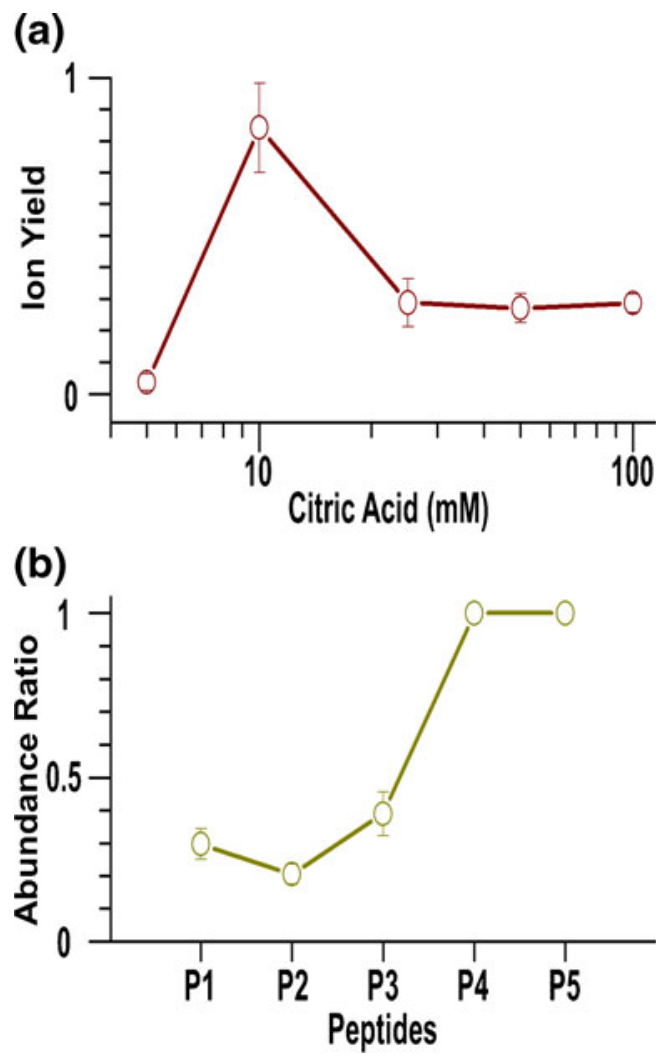

Figure 2. (a) Plots for the effect of the citric acid concentration on the ion yield of insulin (20 pmol). (b) Plots for the effect of the citric acid additives on LDI of five different peptides. The abundance ratio was defined by I(c)/[I(c) + I(w)], where $\mathrm{I}(\mathrm{c})$ denotes the ion abundance obtained with the addition of $10 \mathrm{mM}$ citric acid and $\mathrm{I}(\mathrm{w})$ is that without citric acid. $A$ value higher than 0.5 indicates the enhancement in LDI by citric acid. (P1: bradykinin 1-5, monoisotopic mass= 572.3 Da; P2: angiotensin II, 1045.5 Da; P3: melittin, 2844.8 Da; P4: insulin chain b oxidized, $3493.6 \mathrm{Da}$; P5: insulin, 5729.6 Da)
The LDI-on-SOI method was found to be very sensitive, and it exhibited a good detection limit of greater than $20 \mathrm{fmol}$ for angiotensin II. However, there was no noticeable difference in the performance between the $50 \mathrm{~nm}$ and $100 \mathrm{~nm}$ SOI wafers. Accordingly, the results demonstrate that the SOI wafers with a thin Si layer offer a platform for LDI of thermally labile molecules such as peptides.

\section{Effect of Citric Acid Additives}

Various additives that have been reported to improve the LDI efficiency, including citric acid [14] and citrates [6], were tested. In particular, the addition of citric acid was found to enhance the performance of LDI on SOI wafers. The citric acid greatly extended the detectable mass range, which led to successful ionization of $10 \mathrm{pmol}$ insulin molecules. The use of $10 \mathrm{mM}$ citric acid was found to be

(a)

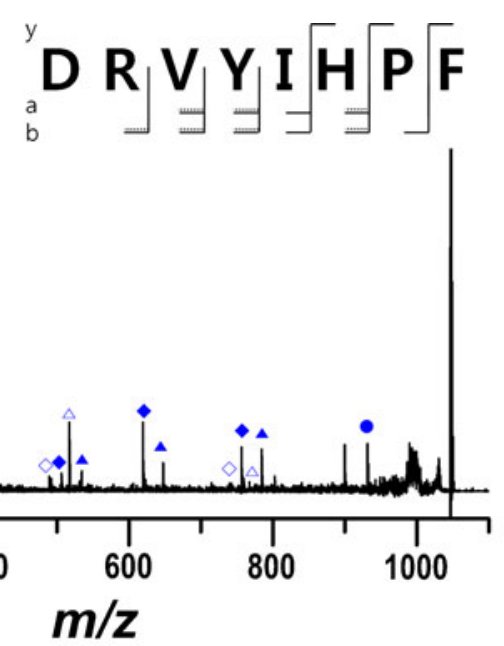

(b)

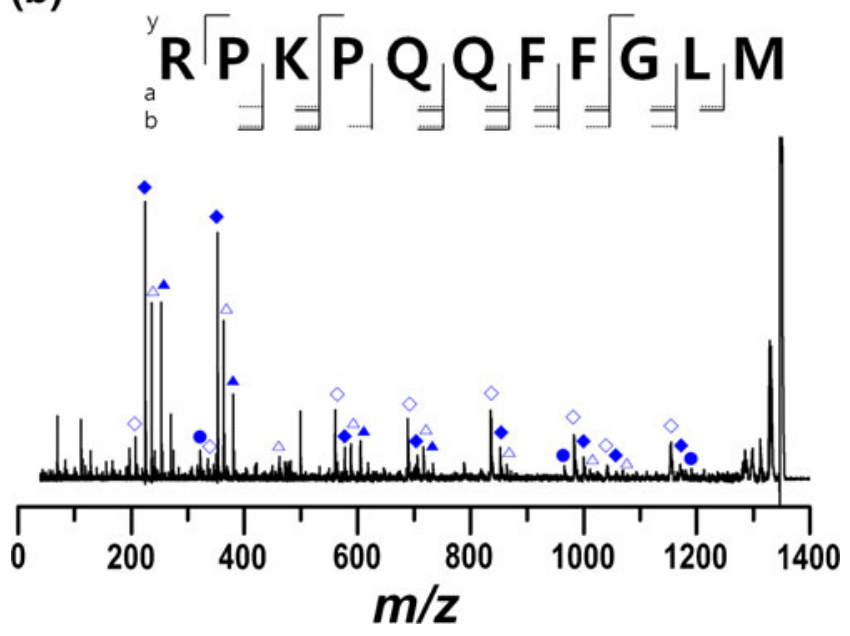

Figure 3. PSD spectra of (a) angiotensin II and (b) substance $\mathrm{P}$, where $\bullet$ : a ions, $\diamond: \mathrm{a}-17$ ions, $\triangleleft$ : b ions, $\Delta$ : b-17 ions, and $\bullet$ : yions. The insets indicate the cleavage sites, where the dotted line indicates the corresponding ions with a loss of $17 \mathrm{Da}$ 
optimal for the peptide ionization (Figure 2a). However, while the presence of citric acid was particularly useful for peptides with large masses ( $>3000 \mathrm{Da})$, the LDI efficiency was slightly degraded for peptides with smaller masses $(<2000 \mathrm{Da})$ (Figure 2b). The addition of ammonium hydrogen citrate (dibasic) [6] and other acids such as trifluoroacetic acid and acetic acid did not result in any noticeable improvement in the LDI performance.

The role of the citric acid additives was studied further with respect to LDI of insulin molecules. The LDI enhancement was evident only when a premixed solution of insulin and citric acid was used as the sample. For example, when the insulin and citric acid solutions were loaded separately (i.e., a sequence of citric acid solution deposition and drying, then of insulin solution deposition) led to very poor ionization. When the solutions were loaded in the reverse order, the formation of insulin ions was rarely observed. The results suggest that the primary role of citric acid is possibly related to the complex formation with analytes, which stabilizes the insulin molecules during the energetic LDI process, rather than coating the surfaces or proton donation.

\section{Post-Source Decay of Peptide Ions for TOF/TOF Mass Spectrometry}

Post-source decay (PSD) is a tandem mass spectrometric technique that is used widely on the MALDI-TOF/TOF platform [15]. In PSD, analyte ions undergo dissociation while flying in the field-free flight region, and their fragments are subsequently mass analyzed by the second TOF mass analyzer. This provides useful information for peptide and protein identification. In MALDI mass spectrometry, PSD of peptide ions is induced through the increase of the internal energy of the ions, which is usually achieved by increasing the MALDI laser power. Similarly, it was found that the increase of laser power also led to pronounced PSD of peptide ions produced by LDI on a-Si surfaces, which supported the notion that laserinduced thermal desorption had a key role in the observed LDI process [7].

The PSD of peptide ions produced from the surfaces of SOI wafers was examined. As shown in Figure 3, the peptide ions of (Figure 3a) angiontensin II and (Figure 3b) Substance P clearly underwent PSD in the TOF/TOF mass spectra, and their fragmentation patterns exhibited essentially the same features produced when using the MALDI method. This result demonstrates that the LDI-on-SOI method can also be applicable to peptide sequencing using TOF/TOF mass spectrometry. The observed PSD created by the LDI-on-SOI process suggests that a thermal mechanism, which is based on laser-induced thermal desorption, is indeed involved in the LDI process, as proposed above.

\section{Conclusions}

LDI of peptides was demonstrated to occur on commercially available SOI wafers. It exhibits good detection sensitivity and mass range for peptides. The use of commercially available wafers as received is beneficial and offers a simple, accessible way of LDI mass spectrometry for thermally labile molecules. In addition, the flat Si surface of the SOI wafer can be used to build various nanobio techniques, which thus provides a possible platform for nanobio sensors, lab-on-a-chip, and similar applications, where the combination with matrix-free mass spectrometric monitoring may offer new research opportunities.

\section{Acknowledgments}

The authors acknowledge support for this research from the Converging Research Center Program through the NRF, funded by MEST (2012K001504). This work was also supported by the Biosignal Analysis Technology Innovation Program (2012-0006053) of MEST via KOSEF.

\section{References}

1. Peterson, D.S.: Matrix-free methods for laser desorption/ionization mass spectrometry. Mass Spectrom. Rev. 26, 19-34 (2007)

2. Wei, J., Buriak, J.M., Siuzdak, G.: Desorption/ionization mass spectrometry on porous silicon. Nature 399, 243-246 (1999)

3. Gulbakan, B., Park, D., Kang, M., Kececi, K., Martin, C.R., Powell, D.H., Tan, W.: Laser desorption ionization mass spectrometry on silicon nanowell arrays. Anal. Chem. 82, 7566-7575 (2010)

4. Lo, C.-Y., Lin, J.-Y., Chen, W.-Y., Chen, C.-T., Chen, Y.-C.: Surfaceassisted laser desorption/ionization mass spectrometry on titania nanotube arrays. J. Am. Soc. Mass Spectrom. 19, 1014-1020 (2008)

5. Nayak, R., Knapp, D.R.: Matrix-free LDI mass spectrometry platform using patterned nanostructured gold thin film. Anal. Chem. 82, 7772$7778(2010)$

6. Chen, W.-T., Chiang, C.-K., Lee, C.-H., Chang, H.-T.: Using surfaceassisted laser desorption /ionization mass spectrometry to detect proteins and protein-protein complexes. Anal. Chem. 84, 1924-1930 (2012)

7. Kim, S.H., Lee, A., Song, J.Y., Han, S.Y.: Laser-induced thermal desorption facilitates post-source decay of peptide ions. J. Am. Soc. Mass Spectrom. 23, 935-941 (2012)

8. Kim, S.H., Park, S.H., Song, J.Y., Han, S.Y.: Observation of peptideion generation by laser-induced surface heating from tungsten silicide surfaces. Mass Spectrom. Lett. 3, 18-20 (2012)

9. Suckau, D., Resemann, A., Schuerenberg, M., Hufnagel, P., Franzen, J., Holle, A.: A novel MALDI LIFT-TOF/TOF mass spectrometer for proteomics. Anal. Bioanal. Chem. 376, 952-965 (2003)

10. Alimpiev, S., Nikiforov, S., Karavanskii, V., Minton, T., Sunner, J.: On the mechanism of laser-induced desorption-ionization of organic compounds from etched silicon and carbon surfaces. J. Chem. Phys. 115, 1891-1901 (2001)

11. Shin, W.J., Shin, J.H., Song, J.Y., Han, S.Y.: Effects of ZnO nanowire length on surface-assisted laser desorption/ionization of small molecules. J. Am. Soc. Mass Spectrom. 21, 989-992 (2010)

12. Daves Jr., G.D.: Mass spectrometry of involatile and thermally unstable molecules. Acc. Chem. Res. 12, 359-365 (1979)

13. Celler, G.K., Cristoloveanu, S.: Frontiers of silicon-on-insulator. $J$ Appl. Phys. 93, 4955-4978 (2003)

14. Duan, J., Linman, M.J., Cheng, Q.: Ultrathin calcinated films on a gold surface for highly effective laser desorption/ionization of biomolecules. Anal. Chem. 82, 5088-5094 (2010)

15. Chaurand, P., Luetzenkirchen, F., Spengler, B.: Peptide and protein identification by matrix-assisted laser desorption ionization (MALDI) and MALDI-post-source decay time-of-flight mass spectrometry. J. Am. Soc. Mass Spectrom. 10, 91-103 (1999) 